\title{
Heart sign in Neurology - A Radiological sign
}

\author{
Sheetal Sajan $D M^{1}$, Reji Thomas $D M^{2}$ \\ 1,2 Department of Neurology, Pushpagiri Institute of Medical Sciences and Research, Tiruvalla, Kerala, \\ India
}

Date of submission: $19^{\text {th }}$ April 2020

Date of acceptance: $3^{\text {rd }}$ June 2020

Date of publication: $12^{\text {th }}$ August 2020

\begin{abstract}
Bilateral medial medullary infarction is a rare stroke syndrome. The common symptoms are quadriparesis, dysarthria and hypoglossal palsy. However, the early diagnosis of bilateral medial medullary infarction may be difficult as it may mimic other conditions like Guillain Barre syndrome and brainstem encephalitis. With the advent of magnetic resonance imaging, it can be easily diagnosed, as the diffusion weighted sequences demonstrate hyperintensity involving bilateral medulla in a heart shaped pattern, described as the "Heart sign". We hereby report a patient who presented with progressive quadriparesis, and dysarthria, progressing to respiratory failure, whose MRI brain showed the characteristic "heart sign" on diffusion sequences and was diagnosed to have bilateral medial medullary infarction.
\end{abstract}

Key words: Bilateral medial medullary infarction, Heart sign, Magnetic resonance imaging

\section{Introduction}

$\mathrm{M}$ edial medullary infarction accounts for only less than $1 \%$ of all cases of infarctions of the brain, and very rarely occurs bilaterally. ${ }^{1}$ The most common symptoms are dysarthria, hypoglossal palsy and quadriplegia. ${ }^{1}$ Early diagnosis remains a challenge, because the clinical presentation can mimic other conditions such as GuillainBarre syndrome (GBS). ${ }^{2}$ The characteristic brain MRI finding described is "heart appearance" in diffusion weighted sequences. ${ }^{3}$ We hereby report a patient who

Access this article online

Website: https://www.nepjol.info/index.php/NJN

DOI: https://doi.org/10.3126/njn.v17i2.30165

HOW TO CITE

Sajan S, Thomas R. Heart sign in Neurology - A Radiological sign. NJNS. 2020; 17(2):48-50

${ }^{1}$ ORCID id: 0000-0003-3269-3828

${ }^{2}$ ORCID id: 0000-0003-3588-2317

Address for correspondence:

Dr. Sheetal Sajan

16 FG, The Edge, Skyline Apartments

Thirumoolapuram- 689115

Thiruvalla, Kerala, India

Contact number: +91-9446417979

E-mail: sheetalrehaan@gmail.com

Copyright (C 2020 Nepalese Society of Neurosurgeons (NESON)

ISSN: 1813-1948 (Print), 1813-1956 (Online)

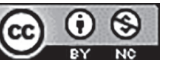

This work is licensed under a Creative Commons Attribution-Non Commercial 4.0 International License. presented with progressive quadriparesis, and dysarthria, progressing to respiratory failure, whose MRI brain showed the characteristic "heart sign" on diffusion sequences and was diagnosed to have bilateral medial medullary infarction.

\section{Case Report}

A 67-year-old gentleman, hypertensive and diabetic, presented with hyper-acute onset of nausea, vomiting and fatigue. He complained of generalized weakness and claimed that he was unable to get out of the bed, as he was feeling dizzy. By the next morning, he complained of weakness of all limbs and had slurred speech. By noon he was noted to have respiratory distress. On examination, he was conscious, higher mental functions were normal and had slurred speech. His heart rate was $80 /$ minute and regular, and blood pressure was 140/90 $\mathrm{mm} \mathrm{Hg}$. Cranial nerve examination was unremarkable. Gag reflex was normal. Motor system examination revealed normal power of neck muscles, hypotonia of all limbs, with grade 3 power in all limbs. All deep tendon reflexes were sluggish, with mute plantar response. Sensory system examination was normal. Few hours later, he developed respiratory paralysis and was intubated and ventilated. The possibilities considered were Guillain Barre syndrome, myasthenic crisis and brainstem encephalitis. MRI brain $(1.5 \mathrm{~T})$ was taken and it showed diffusion restriction in bilateral antero-medial medullary regions in a heart shaped pattern - the characteristic "Heart sign" (Figure 1). Hence the diagnosis of bilateral medial medullary infarction was made. He was initiated on antiplatelets 


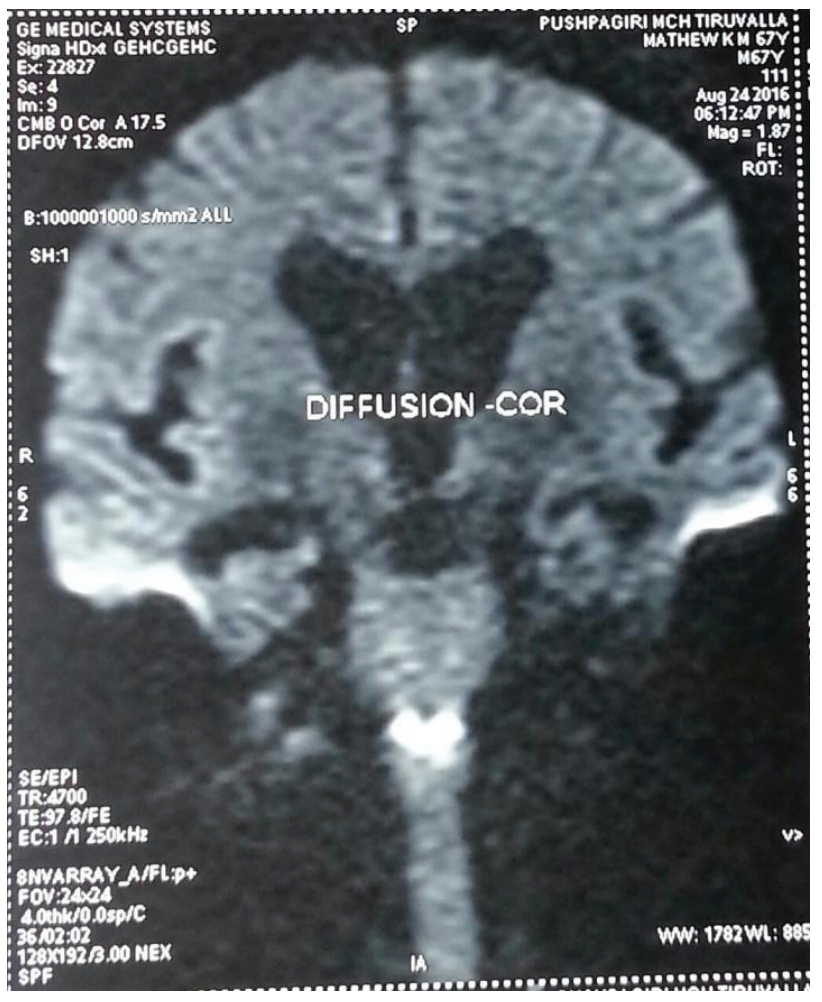

a

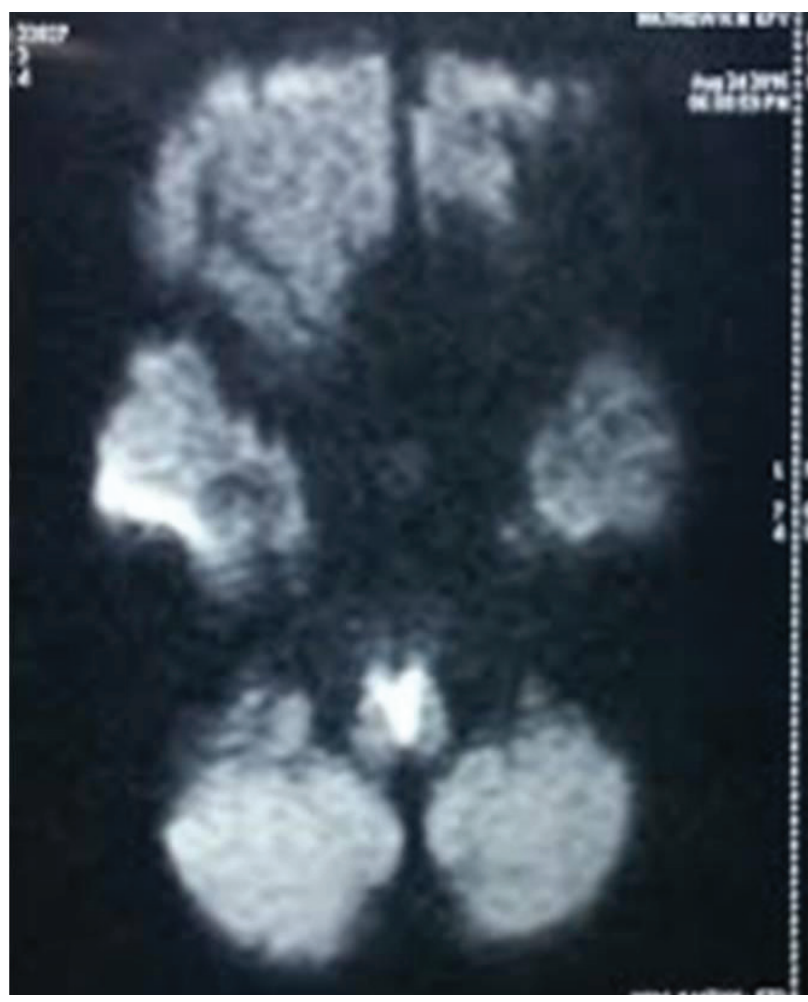

b

Figure 1: Magnetic resonance imaging of the brain - (a) oblique coronal and (b) axial, diffusion weighted sequences showing heart shaped hyperintensity involving bilateral antero-medial medulla - "The Heart sign"

and statin. He remained on endotracheal tube for a week, after which tracheostomy was done. He was weaned off the ventilator by the $2^{\text {nd }}$ week of stay in the intensive care unit, remained conscious, oriented, with normal cranial nerve examination. However, the power in all 4 limbs continued to be grade $1 / 5$ and plantar reflex was bilaterally equivocal.

\section{Discussion}

The first case of bilateral medial medullary infarction was described by Davison and the symptoms described include acute onset quadriparesis, tongue weakness and facial sparing. ${ }^{4}$ Brainstem encephalitis, Guillain-Barre syndrome (GBS), myasthenic crisis, periodic paralysis can present similarly, thereby making the initial diagnosis of this entity difficult. ${ }^{2} \mathrm{Kim}$ et al studied 86 patients with medial medullary infarction, of which bilateral infarction was noted only in 12 (14\%). ${ }^{5}$ In their study, motor dysfunction was the most common symptom, occurring in 78 of 86 patients, with quadriparesis reported in 8 cases $(9.3 \%)$. Sensory dysfunction was noted in $73 \%$ of patients. Tongue deviation was observed only in 12 patients (13.9\%). ${ }^{5}$ Although ipsilateral hypoglossal nerve palsy is one component of the triad of medial medullary infarct, its prevalence has been variably reported as $11 \%$ to $18 \%$ or
$71 \%$ to $82 \%{ }^{6}$ In this patient too, tongue deviation was not observed. Since the hypoglossal nucleus is a cell column of $18 \mathrm{~mm}$ in the medulla, infarction limited to a small portion does not cause hypoglossal nerve palsy. ${ }^{7}$ Bilateral medial medullary infarction carries poor prognosis. Of the 86 patients described by Kim et al, 3 died during admission. ${ }^{5}$

Infarcts involving the medulla oblongata are categorized on the basis of its vascular supply into four territories: anterior-medial territory, anterior-lateral territory, lateral territory, and posterior territory. The "heart appearance" sign is considered to appear when the infarct occurs in the anterior-medial territory and anterior-lateral territory. ${ }^{5}$ In acute bilateral medial medullary infarction, there will be diffusion restriction with low ADC values involving bilateral medial medulla, resulting in the typical "heart sign".

\section{Conclusion}

The early diagnosis of bilateral medial medullary infarction is difficult as it may mimic other conditions like Guillain Barre syndrome and brainstem encephalitis. The demonstration of the 'Heart sign' on magnetic resonance imaging of the brain helps in distinguishing this rare stroke syndrome. 
Conflict of Interest: None

Source(s) of support: None

\section{References}

1. Bassetti C, Bogousslavsky J, Mattle H, Bernasconi A. Medial medullary stroke: report of seven patients and review of the literature. Neurology. 1997;48(4):88290. PMID: 9109872. https://doi.org/10.1212/ wnl.48.4.882

2. Ramakrishnan TCR, Kanagaraju C, Akhtar S, Patel A. Heart in Brain. JCR. 2017; 7:332-4. https://doi. org/10.17659/01.2017.0090

3. Kleinert G, Fazekas F, Kleinert R, Schmidt R, Payer $\mathrm{F}$, Offenbacher $\mathrm{H}$, et al. Bilateral medial medullary infarction: magnetic resonance imaging and correlative histopathologic findings. European Neurology. 1993; 33:74-6. https://doi.org/10.1159/000116906

4. Davison C. Syndrome of the anterior spinal artery of the medulla oblongata. Arch Neurol Psychiatry. 1937; 37:97-107. https://doi.org/10.1001/ archneurpsyc.1937.02260130101007

5. Kim JS, Han YS. Medial medullary infarction: clinical, imaging, and outcome study in 86 consecutive patients. Stroke. 2009; 40(10):3221-5. https://doi. org/10.1161/STROKEAHA.109.559864.

6. Bassetti C, Bogousslavsky J, Mattle H, Bernasconi A. Medial medullary stroke: report of seven patients and review of the literature. Neurology. 1997; 48:882-90. https://doi.org/10.1212/wnl.48.4.882

7. Ho KL, Meyer KR: The medial medullary syndrome. Arch Neurol. 1981; 38:385-7. https://doi. org/10.1001/archneur.1981.00510060087020 